\title{
A Novel Inorganic Sulfur Compound Metabolizing Ferroplasma-Like Population Is Suggested to Mediate Extracellular Electron Transfer
}

\section{OPEN ACCESS}

Edited by:

Christopher Bagwell,

Pacific Northwest National Laboratory

(DOE), United States

Reviewed by:

Jeongdae Im

Kansas State University, United States

Tim Magnuson,

Idaho State University, United States

${ }^{*}$ Correspondence:

Gaofeng Ni

gaofeng.ni@outlook.com

tPresent address:

Domenico Simone,

Bioinformatics Infrastructure, Swedish

University of Agricultural Sciences,

Uppsala, Sweden

Elias Broman,

Department of Ecology, Environment and Plant Sciences, Stockholm

University, Stockholm, Sweden

Specialty section:

This article was submitted to Microbiotechnology, Ecotoxicology and Bioremediation,

a section of the journal

Frontiers in Microbiology

Received: 10 August 2018 Accepted: 16 November 2018 Published: 05 December 2018

Citation:

Ni G, Simone D, Palma D, Broman E, Wu X, Turner S and Dopson M (2018) A Novel Inorganic

Sulfur Compound Metabolizing

Ferroplasma-Like Population is Suggested to Mediate Extracellular Electron Transfer.

Front. Microbiol. 9:2945. doi: 10.3389/fmicb.2018.02945

\section{Gaofeng Ni*, Domenico Simonet, Daniela Palma, Elias Broman ${ }^{\dagger}$, Xiaofen Wu, Stephanie Turner and Mark Dopson}

Centre for Ecology and Evolution in Microbial Model Systems (EEMiS), Linnaeus University, Kalmar, Sweden

Mining and processing of metal sulfide ores produces waters containing metals and inorganic sulfur compounds such as tetrathionate and thiosulfate. If released untreated, these sulfur compounds can be oxidized to generate highly acidic wastewaters [termed 'acid mine drainage (AMD)'] that cause severe environmental pollution. One potential method to remediate mining wastewaters is the maturing biotechnology of 'microbial fuel cells' that offers the sustainable removal of acid generating inorganic sulfur compounds alongside producing an electrical current. Microbial fuel cells exploit the ability of bacterial cells to transfer electrons to a mineral as the terminal electron acceptor during anaerobic respiration by replacing the mineral with a solid anode. In consequence, by substituting natural minerals with electrodes, microbial fuel cells also provide an excellent platform to understand environmental microbemineral interactions that are fundamental to element cycling. Previously, tetrathionate degradation coupled to the generation of an electrical current has been demonstrated and here we report a metagenomic and metatranscriptomic analysis of the microbial community. Reconstruction of inorganic sulfur compound metabolism suggested the substrate tetrathionate was metabolized by the Ferroplasma-like and Acidithiobacilluslike populations via multiple pathways. Characterized Ferroplasma species do not utilize inorganic sulfur compounds, suggesting a novel Ferroplasma-like population had been selected. Oxidation of intermediate sulfide, sulfur, thiosulfate, and adenylylsulfate released electrons and the extracellular electron transfer to the anode was suggested to be dominated by candidate soluble electron shuttles produced by the Ferroplasma-like population. However, as the soluble electron shuttle compounds also have alternative functions within the cell, it cannot be ruled out that acidophiles use novel, uncharacterized mechanisms to mediate extracellular electron transfer. Several populations within the community were suggested to metabolize intermediate inorganic sulfur compounds by multiple pathways, which highlights the potential for mutualistic or symbiotic relationships. This study provided the genetic base for acidophilic microbial fuel cells utilized for the remediation of inorganic sulfur compounds from AMD.

Keywords: bioelectrochemical systems, electricigens, Ferroplasma, Acidithiobacillus, sulfide mineral mining, metagenome assembled genomes, metatranscriptomics 


\section{INTRODUCTION}

The extraction of metals from sulfide ores is a robust industrial process carried out in many countries around the world. However, when wastes from sulfide mineral processing are exposed to water and oxygen they can oxidize and form acid mine drainage (AMD), which is characterized by extreme acidity and high metal content (Huang et al., 2016). If released untreated, AMD can damage the environment disastrously and distress the fauna and flora within (Amisah and Cowx, 2000; Hill et al., 2000; Sola et al., 2004). Bacteria and archaea that thrive under acidic environments catalyze AMD formation and these microorganisms are termed acidophiles $(\mathrm{pH}$ optimum $<5$ ) and extreme acidophiles ( $\mathrm{pH}$ optimum $<3$ ). They are often involved in redox transformations of inorganic sulfur compounds (ISCs) and iron containing minerals (Dopson and Johnson, 2012; Johnson et al., 2012) and they have also been exploited for the removal of acid generating ISCs (Liljeqvist et al., 2011). For instance, Acidithiobacillus caldus and A. ferrivorans are known for chemoautotrophic growth on ferrous iron and/or reduced ISCs (Valdes et al., 2009; Hallberg et al., 2010; Mangold et al., 2011; González et al., 2014). A. ferrivorans is a facultative anaerobe while characterized strains of $A$. caldus require oxygen for growth (Hallberg and Lindström, 1994; Hallberg et al., 2010; González et al., 2014). In addition, archaeal populations such as Ferroplasma are dominant in extremely acidic AMD environments (Edwards et al., 2000) and published isolates are facultative anaerobes that are capable of chemoorganotrophic growth on yeast extract coupled to the reduction of ferric iron (Dopson et al., 2004).

The emerging biotechnology of microbial fuel cells (MFCs) utilize electrochemically active microorganisms to oxidize substrates and transfer the released electrons to the anode (Logan et al., 2006; Rozendal et al., 2008). From the anode, the electrons flow to the cathode where they are consumed during reduction reactions. This electron flow is harvested as an electrical current and can be used to power low-energy demanding devices such as biological oxygen demand sensors (Di Lorenzo et al., 2014) or to generate useful products such as hydrogen gas (Kuntke et al., 2014). As the MFC technology utilizes microbial catalysis to remediate wastewater, it eliminates the need for the addition of chemical catalysts and recovers electrical energy. Therefore, it is considered energy efficient and renewable (Rozendal et al., 2008). Recently, MFCs have evolved into a versatile technology and their integration with extremophilic microorganisms has widened the choice of electron donors, including inorganic sulfur compounds. As a result, possibilities to treat environmental pollutants under extreme conditions have been opened and MFCs and their uses with extremophiles have been reviewed in Dopson et al. (2016). Recent studies have shown that electrical current (maximum $433 \mathrm{~mA} \mathrm{~m}^{-2}$ ) can be generated from the degradation of the ISC tetrathionate $\left(\mathrm{S}_{4} \mathrm{O}_{6}{ }^{2-}\right)$ at $\mathrm{pH} 2.5$ (Sulonen et al., 2015) and ISCs in real wastewater from sulfide mineral processing (Ni et al., 2016). These data suggest that acidophile-integrated MFCs are promising for treating AMD-related wastewaters.

The MFC technology is based on the metabolic capability of microorganisms to deliver electrons derived from metabolism across the cell envelope to reduce extracellular electron acceptors, a process termed 'extracellular electron transfer' (EET) (Lovley, 2008; Rozendal et al., 2008). This is a widely occurring phenomenon, for instance by dissimilatory metal reducing bacteria such as Geobacter and Shewanella that reduce metals such as ferric iron or manganese (Nealson et al., 2002; Shi et al., 2007). Such processes are of global importance for iron and sulfur cycling. Currently, molecular characterization of EET mechanisms is limited to Geobacter, Shewanella, and Pseudomonas, revealing three categories including: (i) Outer membrane $c$-type cytochromes for direct electron transfer. For instance, multi-heme $c$-type cytochromes encoded by genes such as mtrAC, cymA, omcAESBZ, and gspG in Geobacter and Shewanella species were shown to be crucial in carrying out EET to metal oxides or MFC electrodes (Holmes et al., 2006; Shi et al., 2007; Bretschger et al., 2008; Richter et al., 2009). (ii) Redox-active molecules (e.g., menaquinone, riboflavin, and phenazines) that function as electron shuttles. It was shown that the menC gene is involved in menaquinone and quinone intermediate biosynthesis and its absence severely hampers the ability of Shewanella spp. to perform EET (Newman and Kolter, 2000; Myers and Myers, 2004). It was also reported that riboflavin is the key component secreted by Shewanella spp. that utilizes the electrode as an electron acceptor (Marsili et al., 2008). Furthermore, phenazines were reported to function as electron shuttles in the reductive dissolution of minerals and the electron transfer to electrode (Hernandez et al., 2004; Wang et al., 2010). (iii) Conductive pili are capable of direct electron transfer, as it was shown that mutants of Shewanella deficient in the type II secretion system and type IV pilin are severely limited in the current producing capacity (Bretschger et al., 2008; Richter et al., 2009).

Metagenomics is the cultivation-independent sequencing and characterization of DNA from the microbial community that enables the elucidation of the genetic potential of the entire community (Sharon and Banfield, 2013; Hu et al., 2016). Metatranscriptomics is the profiling of gene transcripts from the complete microbial community and can further elucidate the community's response to a specific experimental or environmental stimulus. 'Omics' studies provide a snapshot of the microbial community profile at the time of extraction. Although the presence of mRNA transcripts does not prove a metabolic process is occurring (Rocca et al., 2015), this approach offers a powerful tool to gain biological insights into the processes of interest. For example, metatranscriptomics can be utilized to identify potential EET stimuli-sensitive genes by comparing gene expression at varying EET rates (Ishii et al., 2013). However, a combined metagenomic and metatranscriptomic investigation of EET has not been conducted on an acidophilic MFC anodic microbiome.

Previously, duplicate MFCs with tetrathionate as substrate and carbon dioxide (as bicarbonate) for carbon source were used to treat ISCs at pH 2.5 (Ni et al., 2016). Tetrathionate was chosen as the substrate as it commonly exists in the flotation process water of sulfide ores (Liljeqvist et al., 2011) and it is stable at low $\mathrm{pH}$. The MFCs were inoculated in the anode chamber with an anoxic sediment from a $\mathrm{pH} 2.5-2.7$ AMD stream from the Kristineberg mine, northern Sweden [the AMD conditions 
are described in Liljeqvist et al. (2015)]. Amplification of the $16 \mathrm{~S}$ rRNA genes from the anodic microbial consortium aligned within the Ferroplasmaceae, Acidithiobacillus, Clostridiaceae, and Sulfobacillus (Ni et al., 2016). Generation of electrical current coupled to tetrathionate degradation was confirmed as being biologically mediated based on (i) cyclic voltammetry scans of the biotic operation showed higher current than the abiotic control; (ii) abiotic operation generated electrical current but it decreased more rapidly compared to biotic operation; and (iii) subsequent inoculation of the abiotic system with the same AMD culture used in the MFCs without additional tetrathionate resulted in an increased cell voltage (Ni et al., 2016).

To unravel the molecular evidence for the degradation of tetrathionate coupled to the generation of an electrical current, we investigated the anodic microbial consortia from previously reported duplicate MFCs (Ni et al., 2016), termed sample 'S1' and 'S2' for the biological replicate metagenomes and metatranscriptomes. The anode communities' taxonomic affiliation and metabolic potential were based upon reconstruction of metagenomic-assembled genomes (MAGs). In addition, mRNA transcripts were identified for processes involved in ISC metabolism, EET, inorganic carbon metabolism, and adaptations to conditions within the MFCs. Noticeably, the use of ISCs in the MFC technology under acidophilic conditions is highly novel and molecular EET mechanisms for acidophilic microorganisms are poorly understood.

\section{METHODOLOGY}

\section{DNA, RNA Extraction and High Throughput Sequencing}

Planktonic cells were harvested from the anodic compartment of the duplicate MFCs when they were fed with a synthetic media containing $5 \mathrm{mM}$ tetrathionate (Ni et al., 2016). Biomass for DNA extraction was harvested by filtering the anolyte through a $0.2 \mu \mathrm{m}$ sterile filter (Merck Millipore, United States) when the cell voltage values were 109 and $50 \mathrm{mV}$ from the $\mathrm{S} 1$ and S2 MFCs, respectively. Community DNA was immediately extracted using the PowerWater DNA isolation kit (MO BIO, United States) as instructed by the manufacturer. Cells for RNA extraction were taken from the S1 and S2 anodic compartments when cell voltage values were 41 and $53 \mathrm{mV}$, respectively (Ni et al., 2016). The anolyte was immediately centrifuged at $4^{\circ} \mathrm{C}(10000 \times g$ for $15 \mathrm{~min})$ and the community RNA was extracted according to the manufacturer's instructions using the RNeasy midi kit for isolation of total RNA from bacteria (Qiagen, Germany). DNase clean-up from the extracted RNA samples was using the Turbo DNA-free Kit (Ambion by Life Technologies, United States) according to the manufacturer's protocol. The quality and quantity of the nucleic acid samples were tested on Qubit 2.0 (Life Technologies, United States) and Nanodrop (Thermo Scientific, United States). All DNA and RNA samples were sequenced at SciLifeLab, Stockholm, Sweden. The metagenome sequencing was carried out on two Illumina HiSeq 2500 lanes (HiSeq Control Software 2.2.58/RTA 1.17.21.3) with a $2 \times 151$ bp setup in RapidRun mode.
Metatranscriptome sequencing was carried out on a single lane of a HiSeq 2500 (HiSeq Control Software 2.2.58/RTA 1.18.61) with a $2 \times 126$ bp setup in RapidRun mode without rRNA depletion.

\section{Bioinformatic Analysis of the Metagenome Data}

Bioinformatic analysis of the metagenome sequences (Supplementary File S1) was carried out as described in $\mathrm{Wu}$ et al. (2015). Briefly, the raw reads were trimmed, low quality reads and Illumina adapter sequences removed, reads were assembled into contigs using Ray version 2.3.1 (Boisvert et al., 2010) with various k-mer sizes and were combined using Newbler version 2.6. Since 'binning' of high throughput sequences into metagenome assembled genomes (MAGs) is a crucial step for the downstream analysis, six strategies (see Supplementary File S2) involving the software CONCOCT version 0.3.0 (Alneberg et al., 2014), MetaBAT (Kang et al., 2015), and MyCC (Lin and Liao, 2016) were assessed by the genome quality software CheckM version 1.0.5. Based upon MAG completeness, evaluation of degree of contamination, strain heterogeneity, and microbial diversity (Supplementary File S2); the best strategy for MAG construction was found to be achieved by individual assembly using CONCOCT with length control of input contigs. Taxonomic information within the MAGs was extracted and phylogenomic trees of the MAGs constructed using PhyloPhlAn version 0.99 (Segata et al., 2013) and visualized using Archaeopteryx (Zmasek, 2012). Functional annotation of each MAG was performed with Prokka (Seemann, 2014) and analyzed using the Kyoto Encyclopedia of Genes and Genomes (KEGG) (Kanehisa and Goto, 2000) and the MetaCyc (Caspi et al., 2014) databases.

\section{Bioinformatic Analysis of the Metatranscriptome Data}

The quality of metatranscriptomic datasets from the anodic microbiomes was checked with FastQC (Andrews, 2010) and trimmed using Trimmomatic version 0.32 (Bolger et al., 2014) with the following parameters: LEADING:20, TRAILING:20, SLIDINGWINDOW:4:25, MINLEN:100. For each sample, the rRNA and mRNA read datasets were extracted with SortMeRNA version 2.1b (Kopylova et al., 2012) with default parameters and rRNA databases provided by the authors on the tool github repository ${ }^{1}$. mRNA reads from the two samples were co-assembled to obtain transcripts using default parameters in Trinity version 2.4.0 (Grabherr et al., 2011). The metatranscriptome assembly quality was assessed with the Trinotate pipeline $e^{2}$. Briefly, the representation of full-length reconstructed protein-coding genes was examined by mapping the assembled transcript against the SwissProt database (UniProt Consortium, 2018) using BlastX version 2.6.0+ (Altschul et al., 1990). Read filtering and assembly statistics are reported in

\footnotetext{
${ }^{1}$ https://github.com/biocore/sortmerna

${ }^{2}$ https://trinotate.github.io
} 
Supplementary File S1. The Trinotate pipeline was also used to perform downstream analyses on the assembled transcripts including an estimation of transcript abundance using RSEM version 1.2.29 (Li and Dewey, 2011) and transcript functional annotation using the Gene Ontology (GO) resource (Ashburner et al., 2000). Species activity within the samples was assessed in three complementary procedures: (i) Mapping of mRNA reads to the MAGs with Bowtie2 version 2.2.9 (Langmead and Salzberg, 2012). (ii) Performing a taxonomic assignment of assembled transcripts with Kaiju version 1.5.0 (Menzel et al., 2016) with data reported in Supplementary File S3. (iii) Phylogenetic placement of the rRNA reads. rRNA reads were phylogenetically placed using a reference multiple alignment (RMA) and the related reference phylogenetic tree (RPT) by aligning the reads to the RMA with default parameters in PaPaRa version $2.5^{3}$ before being inserted into the RPT by re-optimization of RPT edge lengths through the Evolutionary Placement Algorithm (EPA) implemented in RAxML [version 8.2.10; (Stamatakis, 2006)]. RAxML-EPA was used with default parameters except the fraction of insertion branches to be evaluated using slow insertions under ML (-G option) which was set to 0.1 , as suggested by the authors. Two RPTs (one archaeal and one bacterial), with the related RMAs and including SSU sequences from Anantharaman et al. (2016) and Hug et al. (2016) were adopted as references to perform the phylogenetic identification. Only phylogenetic placements supported by a likelihood weight ratio of $\geq 0.90$ were retained for further analyses. Abundances of reads associated with each tree node were determined with the guppy v1.1 utility of the pplacer version 1.1.alpha17 package (Matsen et al., 2010) and summarized at the genus level. To investigate if the microbial community contained the sulfite converting APS reductase (encoded by the apr gene), it was attempted to amplify it from the community DNA from the two MFCs by PCR using published and specifically designed primers specific for the aprA or aprB genes (Supplementary File S4).

\section{Accession Number}

The raw metagenome and metatranscriptome sequences are available on the NCBI database with the accession number SRP132763.

\section{RESULTS AND DISCUSSION}

\section{Metagenome Assembled Genomes and RNA Transcripts}

The anodic biomass from the previously described MFCs (Ni et al., 2016) were used for DNA and RNA extraction in this study. For the metagenomic sequencing, 142 and 145 million read pairs were obtained for MFCs S1 and S2, respectively, and for the metatranscriptomic sequencing, 97 and 172 million read pairs were obtained for MFCs S1 and S2, respectively (Supplementary File S1). Reconstruction of near complete MAGs by the tested

${ }^{3}$ https://sco.h-its.org/exelixis/web/software/papara/index.html

${ }^{4}$ https://www.ncbi.nlm.nih.gov/sra/?term=SRP132763 binning strategies and their quality parameters including the level of genome completeness and contamination are detailed in Supplementary File S2. Except for the Ferroplasma-like MAG in S1, the level of genome completeness was greater than $94.9 \%$ across the accepted MAGs and with less than $3.9 \%$ contamination. The transcript annotation and abundance (expressed in transcripts per million, TPMs) in the samples are provided in Supplementary File S3. The Ferroplasma-like MAG in $\mathrm{S} 1 \mathrm{had}$ an increased number of duplicated single copy marker genes used for the estimation of MAG quality (Supplementary Files S5, S6). However, it was retained as it was one of the dominating populations based upon mapped reads and RNA transcripts.

Reconstruction of near complete MAGs by the different binning strategies consistently found the dominant populations to be most similar to Ferroplasma spp., A. caldus, A. ferrivorans, Sulfobacillus thermosulfidooxidans, and Cuniculiplasma divulgatum (Figure 1 and Supplementary File S5). This was in agreement with previous 16S rRNA gene amplicon data that suggested the duplicate anode compartments contained communities that aligned with the Ferroplasmaceae, Acidithiobacillus, Clostridiaceae, Sulfobacillus and unclassified species (Ni et al., 2016). Despite the inocula being from different countries, this community was similar to that from another tetrathionate degrading MFC that amongst other populations, also contained Acidithiobacillus spp. and "Ferroplasma acidarmanus" (Sulonen et al., 2015). Several of the MAGs were most similar to sequenced genomes of cultured species with expected growth characteristics for an ISC metabolizing anode community (Supplementary File S7). For instance, A. ferrivorans and $S$. thermosulfidooxidans are facultative anaerobes capable of oxidizing ISCs (Bridge and Johnson, 1998; Hallberg et al., 2010). However, some characteristics of the phylogenetically closest cultured representatives were surprising to be present in an anaerobic anodic community. For instance, Ferroplasma spp. have not been demonstrated to oxidize ISCs (Golyshina et al., 2000; Dopson et al., 2004) and A. caldus is an obligate aerobe (Hallberg and Lindström, 1994). The fact that the community mediated electrical current generation from the ISCs indicated that potentially novel populations had been selected in the anaerobic anode compartment of the MFC.

Mapping of metagenome reads to the MAGs suggested that Acidithiobacillus-like populations were the most abundant in the community followed by the Ferroplasma-like population, and finally small amounts of S. thermosulfidooxidans-like and C. divulgatum-like populations (Figure 2). Despite not being most abundant in the community according to the mapping of MAGs, the Ferroplasma-like population was the most active at the time of sampling, as suggested by the metatranscriptomic analysis showing that the 99.1 and $97.1 \%$ of the $16 \mathrm{~S}$ rRNA reads were attributed to Ferroplasma in samples S1 and S2, respectively, while 93.0 and $48.6 \%$ of the mRNA TPMs were attributed to the Ferroplasmaceae family in samples $\mathrm{S} 1$ and $\mathrm{S} 2$, respectively (Figure 2). That the Ferroplasma-like population were most active according to the RNA transcripts could have been due to the autotrophic Acidithiobacillus and Sulfobacillus species being limited by carbon dioxide availability. 


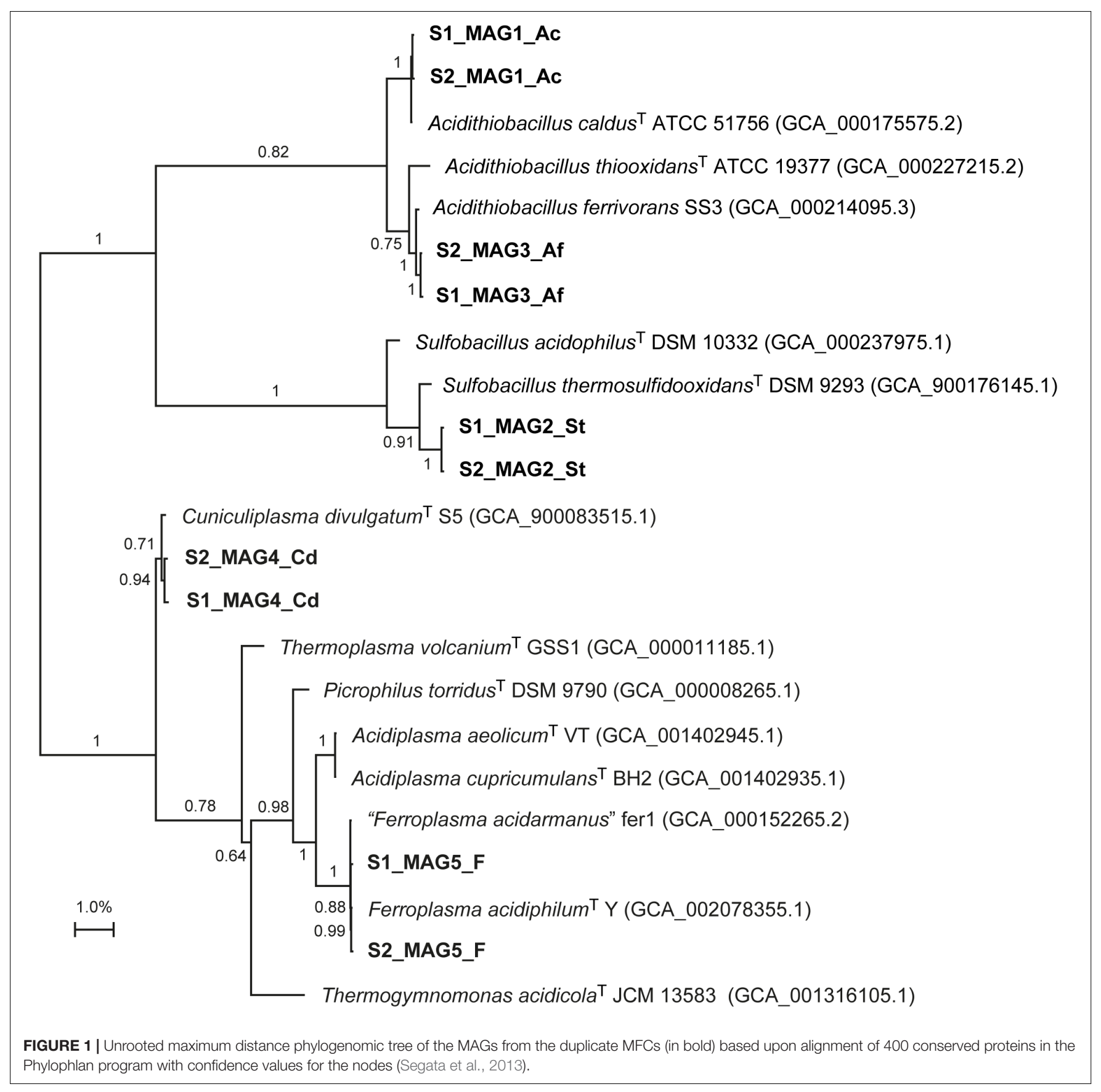

\section{ISC Metabolic Potential Coded Within the MAGs}

Based on the production of sulfate and elemental sulfur, Sulonen et al. (2015) proposed that tetrathionate was disproportionated in a study with a similar MFC set-up. Due to similarities in the selected microbial communities and the observation of elemental sulfur (a product of tetrathionate disproportionation) on the anode surface, the initial step in tetrathionate metabolism in this study was also likely to be disproportionation ( $\mathrm{Ni}$ et al., 2016). However, no genetic evidence for this process in an MFC set-up has been presented. Therefore, we investigated for genes and mRNA expression profiles attributed to the metabolism of tetrathionate and other ISCs (Figures 3, 4). The tet $H$ gene that codes for tetrathionate hydrolase was detected in the A. caldus-like MAGs (Figure 4A and Supplementary File S7), suggesting it was a candidate gene for the initial tetrathionate disproportionation. One product of tetrathionate disproportionation is elemental sulfur that could be metabolized by sulfur oxygenase reductase (encoded by sor; present in all populations except the two C. divulgatumlike MAGs) to hydrogen sulfide and sulfite (Friedrich et al., 2005). Heterodisulfide reductase (encoded by $h d r$ ) converts 


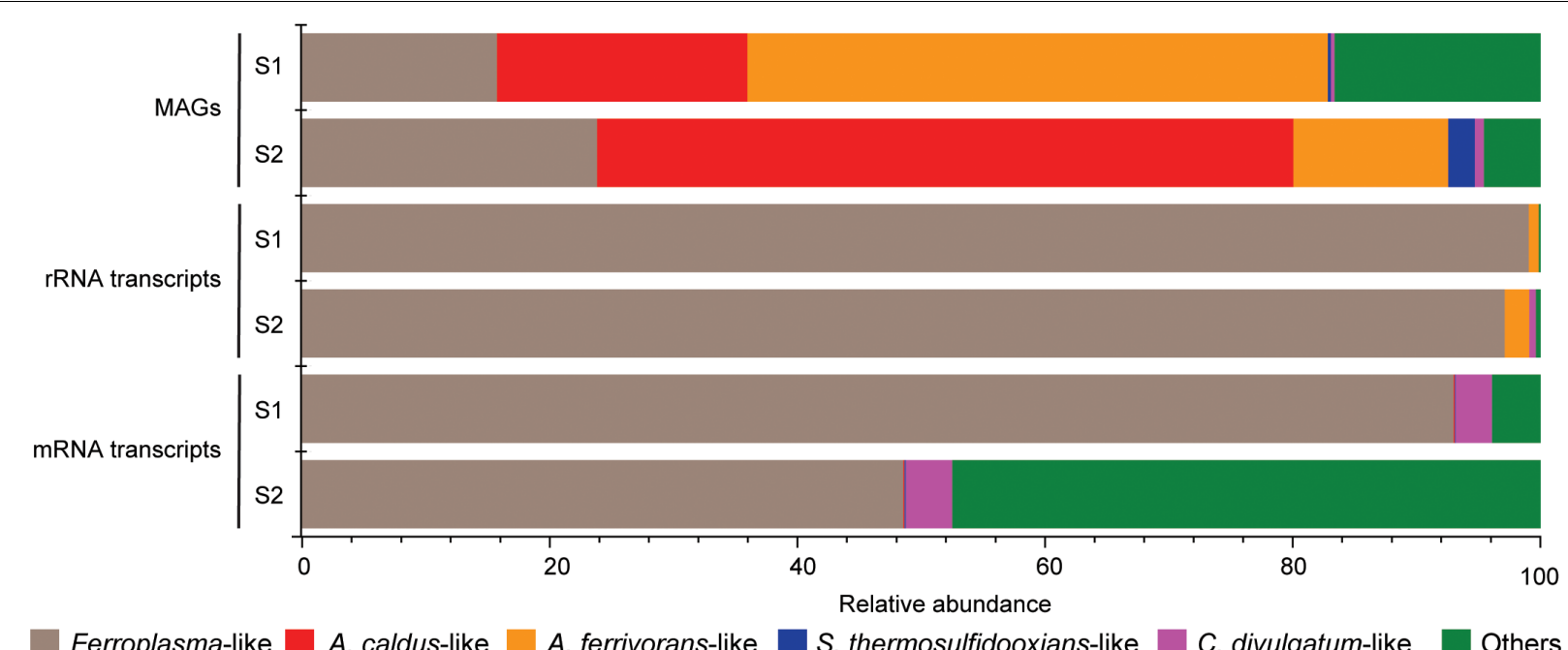

Ferroplasma-like $\square$ A. caldus-like $\square$ A. ferrivorans-like $\square$ S. thermosulfidooxians-like $\square$ C. divulgatum-like $\square$ Others

FIGURE 2 | Relative abundance of the mapped reads that were assigned to the MAGs (top), extracted rRNA reads from the metatranscriptome that were assigned to the MAGs (middle), and mRNA reads mapped to the MAGs (bottom).

the sulfane-sulfur compound glutathione persulfide (GSSH; a more soluble form of elemental sulfur) to sulfite and glutathione (GSH) under anaerobic conditions (Osorio et al., 2013). The $h d r$ gene was present in the Ferroplasma-like and C. divulgatum-like MAGs, suggesting a second possible pathway for elemental sulfur oxidation. The produced sulfide from sulfur oxygenase reductase (encoded by sor) may have been metabolized by the flavocytochrome $c$ sulfide dehydrogenase (Chen et al., 1994) encoded by $f c c B$ present in the Ferroplasmalike, $S$. thermosulfidooxidans-like, and C. divulgatum-like MAGs or the sulfide:quinone oxidoreductase encoded by $s q r$ found in the Ferroplasma-like MAGs. Both the gene products of the sox cluster and the thiosulfate: quinone oxidoreductase encoded by doxDA are predicted to oxidize thiosulfate (Hensen et al., 2006; Mangold et al., 2011; Christel et al., 2016). The genes from the sox cluster were present in one Ferroplasma-like MAG and all bacteria-like MAGs while $\operatorname{doxDA}$ was found in the bacteria-like MAGs. Finally, the produced sulfite was suggested to be oxidized to sulfate in two steps of which one is mediated by APS reductase (encoded by apr), although this gene was absent in this study and has not been identified in acidophiles [e.g., Quatrini et al. (2009) and Mangold et al. (2011)]. The second step is catalyzed by the sat encoded sulfate adenylyltransferase (Quatrini et al., 2009) that was present in all the bacterial MAGs.

The mRNA transcript data (Figure 3) were used to build a model of the community ISC metabolism (Figure 4B). The tet gene for tetrathionate hydrolase had a low number of mRNA transcripts attributed to the Acidithiobacillus-like populations (0 and 12 TPM for S1 and S2, respectively). A potential explanation for this was that the community had depleted the available tetrathionate in the anolyte (as supported by chemical measurement, data not shown) and was oxidizing the ISC intermediates (as supported by the high TPMs for $s q r$ and $h d r$ described below). The starvation of the Acidithiobacillus-like population due to limited tetrathionate may explain its relatively lower activity than the Ferroplasma population. A second explanation could be that the autotrophic A. caldus-like MAGs were limited by the availability of carbon dioxide (detailed in later sections). The majority of the produced elemental sulfur was suggested to be metabolized by heterodisulfide reductase, the product of the $h d r$ gene from the Ferroplasmalike population (1055 and 568 TPM). An alternative to elemental sulfur degradation was disproportionation catalyzed by sulfur oxygenase reductase (product of sor) to form sulfide and sulfite, mRNA transcripts for sor was attributed to the Acidithiobacilluslike populations (12 and 17 TPM). The produced sulfide may then be oxidized by sulfide:quinone oxidoreductase (product of sqr) that had the most abundant transcripts in the metatranscriptome. Based on the mRNA sequence similarity, sqr was attributed to the Ferroplasma population (16780 and 8509 TPM). Finally, thiosulfate and sulfite were potentially metabolized by Acidithiobacillus spp. utilizing the sox and sat gene products, respectively. mRNA transcripts for sox were attributed to A. caldus-like MAGs ( 9 and 14 TPM) while no transcripts were identified for sat from the MAGs of this study. The high number of transcripts coding for ISC metabolism enzymes supports the novel finding that a Ferroplasma-like population can grow via anaerobic sulfur compound oxidation. Overall, tetrathionate was metabolized by multiple members of the community that suggested the potential for syntrophic or mutualistic interactions between these populations. In addition, the oxidation of sulfide, sulfur, thiosulfate, and sulfite released electrons that likely contributed to the generation of electrical current in the MFCs.

\section{Electron Transfer to the Anode}

Based upon inoculated versus abiotic controls, the previously published MFC study (Ni et al., 2016) strongly supported the occurrence of EET to the anode that generated an electrical current. Consequently, we investigated potential mechanisms 


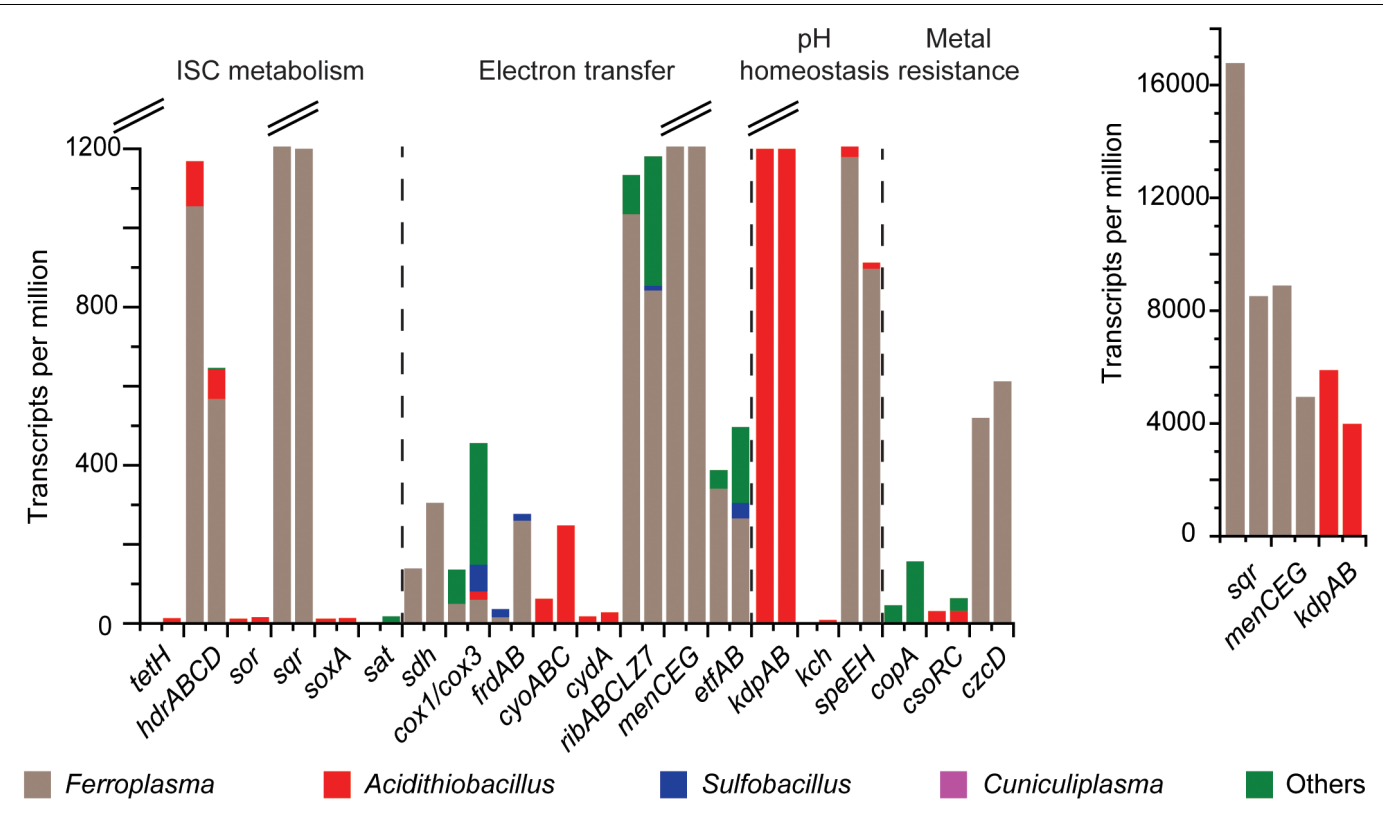

FIGURE 3 | Gene expression profiles for mRNA transcripts annotated (at the genus level) as involved in ISC metabolism, electron transfer to the anode, pH homeostasis, and metal resistance. Genes encode tetrathionate hydrolase (tetH), heterodisulfide reductase (hdr), sulfur oxygenase reductase (sor), sulfide:quinone oxidoreductase (sqr), the sox complex (soxA), ATP sulfurylase (sat), succinate dehydrogenase (sdh), cytochrome $c$ oxidase (cox1/cox3), fumarate reductase (frdAB), cytochrome $\mathrm{bo}_{3}$ ubiquinol oxidase (cyoABC), cytochrome bd-I ubiquinol oxidase (cydA), riboflavin biosynthesis (ribABCLZ7), o-succinylbenzoate synthase (menCEG), electron transfer flavoprotein (etfAB), $\mathrm{K}^{+}$-transporting ATPase ( $\left.k d p A B\right)$, voltage-gated $\mathrm{K}^{+}$channel (kch), spermidine synthase (speEH), Cu-exporting P-type ATPase (copA), $\mathrm{Cu}(\mathrm{l})$ transcriptional repressor (csoRC) and $\mathrm{Cd} / \mathrm{Co} / \mathrm{Zn} \mathrm{H} \mathrm{H}^{+} \mathrm{K}^{+}$antiporter (czcD). The left-hand bar is for MFC S1 and the right for sample MFC S2. The $y$-axis was split at 1200 TPM and the inset gives total values for sqr, menCEG, and $k d p A B$.

for EET based on the metagenomic and metatranscriptomic data. All the MAGs contained a suite of genes attributed to the standard components of respiration including $\mathrm{NADH}$ dehydrogenase, succinate dehydrogenase, fumarate reductase, and quinone biosynthesis indicating the presence of electron transfer chains and the ability of the microbial consortium to obtain energy via oxidative phosphorylation (Supplementary File S8). As tetrathionate simultaneously acted as an electron acceptor and donor during disproportionation, the oxidation of the ISC intermediates (e.g., sulfide, sulfur, thiosulfate, and sulfite) released electrons that are transferred to the quinone pool (Dopson and Johnson, 2012). Since electrical current was produced, the microorganisms performed EET on at least a portion of electrons from the oxidation of ISC to the anode as the final electron acceptor.

No genes coding for outer membrane bound multi-heme $c$-type cytochromes were detected in any of the MAGs. Instead, genes encoding the synthesis and secretion of redox shuttles were identified in both the archaeal and bacterial MAGs, including riboflavin biosynthesis proteins and $\mathrm{O}$-succinylbenzoate synthase encoded by menC (Supplementary File S8). Although they have other functions within the cell (Wissenbach et al., 1990; Vitreschak et al., 2002), the high number of mRNA transcripts for menaquinone biosynthesis including menC (5450 and 3110 TPM for S1 and S2, respectively) followed by menE (3232 and 1738 TPM) and menG (208 and 102 TPM), as well as for the riboflavin biosynthesis genes ribABLZ (1022 and 962 TPM) from the Ferroplasma-like population suggested they mediated EET via soluble electron shuttles. Although the known EET mediating pilA and pilD genes were lacking, other components of the type-IV pili formation genes were identified in all the bacterialike populations (Supplementary File S8), suggesting that these populations have the genetic potential to conserve energy and carry out EET via conductive pili (Bretschger et al., 2008; Richter et al., 2009). However, mRNA transcripts coding for these genes were not identified. The cultured strain of $C$. divulgatum has the ability to grow anaerobically (Golyshina et al., 2016) and the $C$. divulgatum-like MAGs contained genes annotated for riboflavin biosynthesis proteins expressed at 215 and 345 TPM for $\mathrm{S} 1$ and S2, respectively, and $\mathrm{O}$-succinylbenzoate synthase menC expressed at 22 and 65 TPM for S1 and S2, respectively. This suggested that the $C$. divulgatum-population might also carry out EET via soluble electron shuttles. As the C. divulgatumlike MAGs lack genes for ISC oxidation, it is possible that they grew on organic carbon excreted by acidophiles (Nancucheo and Johnson, 2010) and utilize soluble electron shuttles to carry out EET as an energy conservation strategy. Finally, it cannot be categorically ruled out that a small amount of oxygen leaked into the MFC (such as through the rubber tubing) and this potential alternative electron acceptor could have contributed to the low Coulombic efficiency in the MFCs (Ni et al., 2016). However, aerobic energy conservation was unlikely as seen by lack of mRNA transcripts for sor, an enzyme that requires molecular oxygen to oxidize elemental sulfur; as well as the low gene transcripts for cytochrome $c$ oxidase for oxygen uptake was limited to just MFC S2 (Supplementary File S3). 

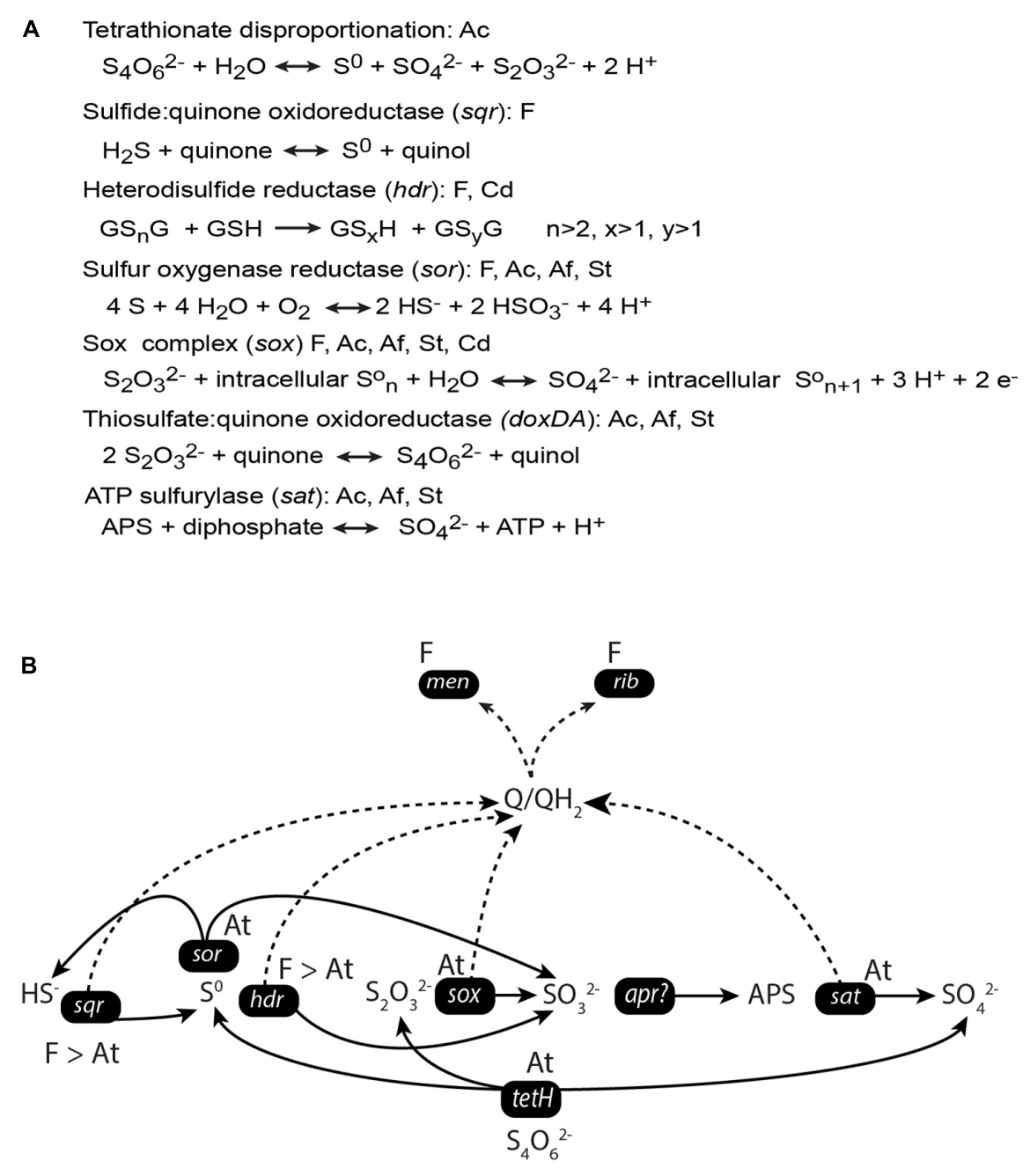

FIGURE 4 | Metabolic potential coded in the MAGs (at the species level) for ISC metabolism in the anode compartments of the duplicate MFCs (A) and mRNA transcripts (at the genus level) for the intermediate steps (B). The model (B) depicts the genera the genes have been assigned to, the gene names, and the relative number of transcripts assigned to the genera as a greater than sign. The solid line indicates the flow of metabolites, the dotted line represents the flow of electrons. Equations are from MetaCyc (Caspi et al., 2014) except for heterodisulfide reductase (hdr) that was adjusted from Rohwerder and Sand (2003). Abbreviations in part (A): F, Ferroplasma-like; Cd, C. divulgatum-like; Ac, A. caldus-like; Af, A. ferrivorans-like; and St, S. thermosulfidooxidans-like populations. Abbreviations in part (B): F, Ferroplasma genus and At, Acidithiobacillus genus.

Evidence for characterized direct EET via outer membrane $c$-type cytochromes is lacking. Although mRNA transcripts for conductive pili formation were not detected, all the bacteria-like populations contained genes encoding type-IV pili formation, offering genomic capacity for an alternative electrode respiration mechanism. mRNA transcripts supported that the large majority of the EET was potentially mediated by soluble shuttles such as menaquinone and riboflavin produced by members of the Ferroplasma genus. Furthermore, it is possible that uncharacterized mechanisms contributed to EET from the acidophiles in this study. It is noteworthy that several known EET mechanisms from neutrophilic populations, e.g., Shewanella and Geobacter were not detected and this highlights the need to investigate EET mechanisms in acidophiles from both MFCs and natural environments.

\section{Carbon Fixation Genes Within the MAGs}

Mining process and waste waters are typically very low in organic carbon (Nitschke and Bonnefoy, 2016) and the MFC communities were required to fix carbon dioxide for autotrophic growth (Figure 2 and Supplementary File S9). The Ferroplasmalike plus the $C$. divulgatum-like MAGs lacked genes for known carbon dioxide fixation pathways and these populations likely grew chemoorganotrophically. This has been shown in a mutualistic interaction whereby autotrophic acidophiles excrete organic carbon that supports chemoorganotrophic growth while chemoorganotrophic populations remove excess organic carbon toxic to autotrophic acidophiles (Slonczewski et al., 2009; Nancucheo and Johnson, 2010). The Acidithiobacilluslike MAGs contained genes attributed to the Calvin-BensonBassham (CBB) cycle and carboxysome formation while 
the S. thermosulfidooxidans-like MAGs contained $c b b$ genes suggesting they are capable of fixing carbon dioxide. The lack of mRNA transcripts encoding carbon fixation (Supplementary File S3) suggested that the carbon dioxide concentration [replenished by carbonate addition in the fed batch MFCs (Ni et al., 2016)] was limiting at the time of cell harvest for metatranscriptomics. This can potentially also explain the lower activity of the Acidithiobacillus-like population compared to the Ferroplasma-like population as suggested by the RNA transcript abundance data.

\section{Adaptation to Low-pH Mining Process and Wastewaters}

The anodic microbial consortium originated from an AMD environment characterized by low $\mathrm{pH}$ and high metal content. To thrive in such environments, acidophilic microorganisms have developed $\mathrm{pH}$ homeostasis and metal resistance mechanisms.

Acidophile $\mathrm{pH}$ homeostatic mechanisms include membranes resistant to proton influx; an internal positive membrane potential suggested to be generated by potassium ions that inhibits proton influx; the utilization of primary and secondary proton pumps as well as proton consuming reactions; and reduced cell permeability [Supplementary File S10, reviewed in Slonczewski et al. (2009), Zammit and Watkin (2016)]. The need for acidophiles to maintain $\mathrm{pH}$ homeostasis in MFCs has a negative effect on cell voltage that lowers power generation and reduced Coulombic efficiency [reviewed in Dopson et al. (2016)]. All of the MAGs contained genes suggested to code for a $k d p \quad \mathrm{~K}^{+}$-transporting ATPase; the Ferroplasma-like, the Acidithiobacillus-like, and the C. divulgatum-like MAGs had a voltage-gated potassium channel kch gene; and the A. caldus-like MAGs had a low affinity $\mathrm{K}^{+}$kup transporter. In contrast, the Ferroplasmalike MAG had a pH-gated $k c s A$ potassium channel and the C. divulgatum-like plus the Ferroplasma-like MAGs had a $\mathrm{Na}^{+} / \mathrm{H}^{+}$antiporter nhaG gene. In addition, the bacterialike MAGs contained one or several $\mathrm{Na}^{+} / \mathrm{H}^{+}$antiporter genes and all except the "Ferroplasma"-like MAGs had an $\mathrm{H}^{+} / \mathrm{Cl}^{-}$exchange transporter $c l c A$ gene. Proton consuming reactions include glutamate decarboxylase (Gut et al., 2006) and one or more of the gadABC genes were identified in all of the MAGs. Spermidine decreases cell permeability via porins (Samartzidou et al., 2003) and all except one C. divulgatum-like MAG had a gene assigned as spermidine synthase (speE) while the two S. thermosulfidooxidans-like MAGs had the most spermidine/putrescine related genes. The mRNA transcripts suggested that the cells were not under large $\mathrm{pH}$ stress as only mRNA transcripts were identified for glutamate decarboxylase and potassium transporters in the Acidithiobacillus and Ferroplasma genera along with speE in Ferroplasma (Figure 3).

Mining waters typically contain high metal concentrations requiring the microbial community to be resistant (Dopson and Holmes, 2014) and therefore, the potential metal and metalloid tolerance systems encoded in the MAGs were investigated (Supplementary File S10). All of the reconstructed
MAGs contained a minimum of $\operatorname{arsRB}$ (in the A. calduslike MAGs) to the complete arsRBCAD set of genes in the S. thermosulfidooxidans-like MAGs suggesting the strains were at least resistant to arsenate (Rosen, 1999). These results were in agreement with characterized strains of " $F$. acidarmanus" (Baker-Austin et al., 2007), A. caldus (Dopson et al., 2001), and S. thermosulfidooxidans (Guo et al., 2014). All of the bacterial MAGs contained either copA or cus genes coding for divalent copper and copper/silver resistance, respectively [reviewed in Dopson and Holmes (2014)]. In addition, the A. calduslike and S. thermosulfidooxidans-like MAGs contained genes attributed to the cso mediated monovalent copper resistance system. The Acidithiobacillus-like MAGs had the most gene copies attributed to the cadmium/cobalt/zinc resistance $c z c$ system. The diverse metal resistance systems in the MAGs are typical of acidophiles inhabiting AMD and reflected the complex multi-metal ore containing zinc, copper, lead, gold, and silver from where the steam flowed from which the inoculum was sampled. That three of the metal resistance related mRNA transcripts were for regulators suggested the cells were not under heavy metal stress in the MFCs containing synthetic media lacking high concentrations of metals (Figure 3).

\section{CONCLUSION}

Microbe-mineral interactions are fundamental to understand the cycling of major elements on earth and a key process is microbial EET for reduction of oxidized minerals. By replacing natural minerals with electrodes, MFCs are a good platform to understand these processes. Here we report a novel Ferroplasma-like population that was suggested to be the most active among the MFC anodic microbial community and metabolized ISCs as well as producing mRNA transcripts encoding redox-active molecules to potentially mediate EET. In addition, the genetic potential and RNA transcript data suggested several populations mediated tetrathionate metabolism. This also suggested symbiotic or mutualistic interactions within the community to carry out multi-species metabolism of ISCs, potential use of soluble electron shuttles, and production of organic carbon by autotrophs that supported heterotrophs. Our findings provided new insights in metabolic capabilities of acidophilic microorganisms and their lifestyle in an engineered environment; elucidated the biological functions of possible future industrial-scale MFCs generating electrical current and remediating mining wastewater as a renewable and energyefficient approach; and called for future research for EET mechanisms in acidophiles.

\section{AUTHOR CONTRIBUTIONS}

MD designed the study. GN and ST carried out the laboratory work. GN, DS, DP, EB, and XW carried out bioinformatic analysis and data analysis. GN, DS, ST, and MD drafted the manuscript. All authors commented and approved the manuscript for publication. 


\section{FUNDING}

This research leading to results has received funding from the European Union Seventh Framework Programme (FP7/2012-2016) under grant agreement number 282970 (BioElectroMET).

\section{ACKNOWLEDGMENTS}

We thank the BioElectroMET consortium members for comments on the manuscript. Sequencing was carried out at

\section{REFERENCES}

Alneberg, J., Bjarnason, B. S., de Bruijn, I., Schirmer, M., Quick, J., Ijaz, U. Z., et al. (2014). Binning metagenomic contigs by coverage and composition. Nat. Methods 11, 1144-1146. doi: 10.1038/nmeth.3103

Altschul, S. F., Gish, W., Miller, W., Myers, E. W., and Lipman, D. J. (1990). Basic local alignment search tool. J. Mol. Biol. 215, 403-410. doi: 10.1016/S00222836(05)80360-2

Amisah, S., and Cowx, I. G. (2000). Impacts of abandoned mine and industrial discharges on fish abundance and macroinvertebrate diversity of the upper River Don in South Yorkshire, UK. J. Freshw. Ecol. 15, 237-250. doi: 10.1080/ 02705060.2000.9663741

Anantharaman, K., Brown, C. T., Hug, L. A., Sharon, I., Castelle, C. J., Probst, A. J., et al. (2016). Thousands of microbial genomes shed light on interconnected biogeochemical processes in an aquifer system. Nat. Commun. 7:13219. doi: $10.1038 /$ ncomms13219

Andrews, S. (2010). FastQC: a Quality Control Tool for High Throughput Sequence Data. Available at: http://www.bioinformatics.babraham.ac.uk/projects/ fastqc

Ashburner, M., Ball, C. A., Blake, J. A., Botstein, D., Butler, H., Cherry, J. M., et al. (2000). Gene ontology: tool for the unification of biology. The Gene Ontology Consortium. Nat. Genet. 25, 25-29. doi: 10.1038/75556

Baker-Austin, C., Dopson, M., Wexler, M., Sawers, R. G., Stemmler, A., Rosen, B. P., et al. (2007). Extreme arsenic resistance by the acidophilic archaeon 'Ferroplasma acidarmanus' Fer1. Extremophiles 11, 425-434. doi: 10.1007/ s00792-006-0052-Z

Boisvert, S., Laviolette, F., and Corbeil, J. (2010). Ray: simultaneous assembly of reads from a mix of high-throughput sequencing technologies. J. Comput. Biol. 17, 1519-1533. doi: $10.1089 / \mathrm{cmb} .2009 .0238$

Bolger, A. M., Lohse, M., and Usadel, B. (2014). Trimmomatic: a flexible trimmer for Illumina sequence data. Bioinformatics 30, 2114-2120. doi: 10. 1093/bioinformatics/btu170

Bretschger, O., Obraztsova, A., Sturm, C. A., Chang, I. S., Gorby, Y. A., Reed, S. B., et al. (2008). Current production and metal oxide reduction by Shewanella oneidensis MR-1 wild type and mutants. Appl. Environ. Microbiol. 74, 553-553. doi: 10.1128/AEM.02560-07

Bridge, T. A. M., and Johnson, D. B. (1998). Reduction of soluble iron and reductive dissolution of ferric iron-containing minerals by moderately thermophilic iron-oxidizing bacteria. Appl. Environ. Microbiol. 64, 2181-2186.

Caspi, R., Altman, T., Billington, R., Dreher, K., Foerster, H., Fulcher, C. A., et al. (2014). The MetaCyc database of metabolic pathways and enzymes and the BioCyc collection of Pathway/Genome Databases. Nucleic Acids Res. 42, D459-D471. doi: 10.1093/nar/gkt1103

Chen, Z. W., Koh, M., Van Driessche, G., Van Beeumen, J. J., Bartsch, R. G., Meyer, T. E., et al. (1994). The structure of flavocytochrome $c$ sulfide dehydrogenase from a purple phototrophic bacterium. Science 266, 430-432. doi: 10.1126/ science.7939681

Christel, S., Fridlund, J., Buetti-Dinh, A., Watkin, E. L., and Dopson, M. (2016). RNA transcript sequencing reveals inorganic sulfur compound oxidation pathways in the acidophile Acidithiobacillus ferrivorans. FEMS Microbiol. Lett. 363:fnw057. doi: 10.1093/femsle/fnw057 the National Genomics Infrastructure hosted by Science for Life Laboratory. Bioinformatic computations were performed on resources provided by SNIC through the Uppsala Multidisciplinary Center for Advanced Computational Science (UPPMAX) under project b2013127.

\section{SUPPLEMENTARY MATERIAL}

The Supplementary Material for this article can be found online at: https://www.frontiersin.org/articles/10.3389/fmicb. 2018.02945/full\#supplementary-material

Di Lorenzo, M., Thomson, A. R., Schneider, K., Cameron, P. J., and Ieropoulos, I. (2014). A small-scale air-cathode microbial fuel cell for on-line monitoring of water quality. Biosens. Bioelectron. 62, 182-188. doi: 10.1016/j.bios.2014.06.050 Dopson, M., Baker-Austin, C., Hind, A., Bowman, J. P., and Bond, P. L. (2004). Characterization of Ferroplasma isolates and Ferroplasma acidarmanus sp. nov., extreme acidophiles from acid mine drainage and industrial bioleaching environments. Appl. Environ. Microbiol. 70, 2079-2088. doi: 10.1128/AEM.70. 4.2079-2088.2004

Dopson, M., and Holmes, D. S. (2014). Metal resistance in acidophilic microorganisms and its significance for biotechnologies. Appl. Microbiol. Biotechnol. 98, 8133-8144. doi: 10.1007/s00253-014-5982-2

Dopson, M., and Johnson, D. B. (2012). Biodiversity, metabolism and applications of acidophilic sulfur- metabolizing micro-organisms. Environ. Microbiol. 14, 2620-2631. doi: 10.1111/j.1462-2920.2012.02749.x

Dopson, M., Lindström, E. B., and Hallberg, K. B. (2001). Chromosomally encoded arsenical resistance of the moderately thermophilic acidophile Acidithiobacillus caldus. Extremophiles 5, 247-255. doi: 10.1007/s007920100196

Dopson, M., Ni, G., and Sleutels, T. H. (2016). Possibilities for extremophilic microorganisms in microbial electrochemical systems. FEMS Microbiol. Rev. 40, 164-181. doi: 10.1093/femsre/fuv044

Edwards, K. J., Bond, P. L., Gihring, T. M., and Banfield, J. F. (2000). An archaeal iron-oxidizing extreme acidophile important in acid mine drainage. Science 287, 1796-1799. doi: 10.1126/science.287.5459.1796

Friedrich, C. G., Bardischewsky, F., Rother, D., Quentmeier, A., and Fischer, J. (2005). Prokaryotic sulfur oxidation. Curr. Opin. Microbiol. 8, 253-259. doi: 10.1016/j.mib.2005.04.005

Golyshina, O. V., Lunsdorf, H., Kublanov, I. V., Goldenstein, N. I., Hinrichs, K. U., and Golyshin, P. N. (2016). The novel extremely acidophilic, cell-walldeficient archaeon Cuniculiplasma divulgatum gen. nov., sp. nov. represents a new family, Cuniculiplasmataceae fam. nov., of the order Thermoplasmatales. Int. J. Syst. Evol. Microbiol. 66, 332-340. doi: 10.1099/ijsem.0.000725

Golyshina, O. V., Pivovarova, T. A., Karavaiko, G. I., Kondrat'eva, T. F., Moore, E. R. B., Abraham, W. R., et al. (2000). Ferroplasma acidiphilum gen. nov., sp. nov., an acidophilic, autotrophic, ferrous-iron-oxidizing, cell-wall-lacking, mesophilic member of the Ferroplasmaceae fam. nov., comprising a distinct lineage of the Archaea. Int. J. Syst. Evol. Microbiol. 50, 997-1006. doi: 10.1099/ 00207713-50-3-997

González, C., Yanquepe, M., Cardenas, J. P., Valdes, J., Quatrini, R., Holmes, D. S., et al. (2014). Genetic variability of psychrotolerant Acidithiobacillus ferrivorans revealed by (meta)genomic analysis. Res. Microbiol. 165, 726-734. doi: 10.1016/ j.resmic.2014.08.005

Grabherr, M. G., Haas, B. J., Yassour, M., Levin, J. Z., Thompson, D. A., Amit, I., et al. (2011). Full-length transcriptome assembly from RNA-Seq data without a reference genome. Nat. Biotechnol. 29, 644-652. doi: 10.1038/nbt.1883

Guo, X., Yin, H., Liang, Y., Hu, Q., Zhou, X., Xiao, Y., et al. (2014). Comparative genome analysis reveals metabolic versatility and environmental adaptations of Sulfobacillus thermosulfidooxidans strain ST. PLoS One 9:e99417. doi: 10.1371/ journal.pone.0099417

Gut, H., Pennacchietti, E., John, R. A., Bossa, F., Capitani, G., De Biase, D., et al. (2006). Escherichia coli acid resistance: $\mathrm{pH}$-sensing, activation by chloride and autoinhibition in GadB. EMBO J. 25, 2643-2651. doi: 10.1038/sj.emboj.7601107 
Hallberg, K. B., Gonzalez-Toril, E., and Johnson, D. B. (2010). Acidithiobacillus ferrivorans, sp. nov.; facultatively anaerobic, psychrotolerant iron-, and sulfuroxidizing acidophiles isolated from metal mine-impacted environments. Extremophiles 14, 9-19. doi: 10.1007/s00792-009-0282-y

Hallberg, K. B., and Lindström, E. B. (1994). Characterization of Thiobacillus caldus, sp. nov., a moderately thermophilic acidophile. Microbiology 140, 34513456. doi: 10.1099/13500872-140-12-3451

Hensen, D., Sperling, D., Truper, H. G., Brune, D. C., and Dahl, C. (2006). Thiosulphate oxidation in the phototrophic sulphur bacterium Allochromatium vinosum. Mol. Microbiol. 62, 794-810. doi: 10.1111/j.1365-2958.2006. 05408.x

Hernandez, M. E., Kappler, A., and Newman, D. K. (2004). Phenazines and other redox-active antibiotics promote microbial mineral reduction. Appl. Environ. Microbiol. 70, 921-928. doi: 10.1128/AEM.70.2.921-928.2004

Hill, B. H., Willingham, W. T., Parrish, L. P., and McFarland, B. H. (2000). Periphyton community responses to elevated metal concentrations in a Rocky Mountain stream. Hydrobiologia 428, 161-169. doi: 10.1023/A:1004028318542

Holmes, D. E., Chaudhuri, S. K., Nevin, K. P., Mehta, T., Methe, B. A., Liu, A., et al. (2006). Microarray and genetic analysis of electron transfer to electrodes in Geobacter sulfurreducens. Environ. Microbiol. 8, 1805-1815. doi: 10.1111/j. 1462-2920.2006.01065.x

Hu, P., Tom, L., Singh, A., Thomas, B. C., Baker, B. J., Piceno, Y. M., et al. (2016). Genome-resolved metagenomic analysis reveals roles for candidate phyla and other microbial community members in biogeochemical transformations in oil reservoirs. $m$ Bio 7:e01669-15. doi: 10.1128/mBio.01669-15

Huang, L. N., Kuang, J. L., and Shu, W. S. (2016). Microbial ecology and evolution in the acid mine drainage model system. Trends Microbiol. 24, 581-593. doi: 10.1016/j.tim.2016.03.004

Hug, L. A., Baker, B. J., Anantharaman, K., Brown, C. T., Probst, A. J., Castelle, C. J., et al. (2016). A new view of the tree of life. Nat. Microbiol. 1:16048. doi: $10.1038 /$ nmicrobiol.2016.1048

Ishii, S., Suzuki, S., Norden-Krichmar, T. M., Tenney, A., Chain, P. S., Scholz, M. B., et al. (2013). A novel metatranscriptomic approach to identify gene expression dynamics during extracellular electron transfer. Nat. Commun. 4:1601. doi: 10.1038/ncomms2615

Johnson, D. B., Kanao, T., and Hedrich, S. (2012). Redox transformations of iron at extremely low pH: fundamental and applied aspects. Front. Microbiol. 3:96. doi: $10.3389 /$ fmicb.2012.00096

Kanehisa, M., and Goto, S. (2000). KEGG: Kyoto encyclopedia of genes and genomes. Nucleic Acids Res. 28, 27-30. doi: 10.1093/nar/28.1.27

Kang, D. D., Froula, J., Egan, R., and Wang, Z. (2015). MetaBAT, an efficient tool for accurately reconstructing single genomes from complex microbial communities. PeerJ 3:e1165. doi: 10.7717/peerj.1165

Kopylova, E., Noe, L., and Touzet, H. (2012). SortMeRNA: fast and accurate filtering of ribosomal RNAs in metatranscriptomic data. Bioinformatics 28, 3211-3217. doi: 10.1093/bioinformatics/bts611

Kuntke, P., Sleutels, T., Saakes, M., and Buisman, C. (2014). Hydrogen production and ammonium recovery from urine by a Microbial Electrolysis Cell. Int. J. Hydrogen Energy 39, 4771-4778. doi: 10.1016/j.ijhydene.2013.10.089

Langmead, B., and Salzberg, S. L. (2012). Fast gapped-read alignment with Bowtie 2. Nat. Methods 9, 357-359. doi: 10.1038/nmeth.1923

Li, B., and Dewey, C. N. (2011). RSEM: accurate transcript quantification from RNA-Seq data with or without a reference genome. BMC Bioinformatics 12:323. doi: 10.1186/1471-2105-12-323

Liljeqvist, M., Ossandon, F. J., González, C., Rajan, S., Stell, A., Valdes, J., et al. (2015). Metagenomic analysis reveals adaptations to a cold-adapted lifestyle in a low-temperature acid mine drainage stream. FEMS Microbiol. Ecol. 91:fiv011. doi: 10.1093/femsec/fiv1011

Liljeqvist, M., Sundkvist, J.-E., Saleh, A., and Dopson, M. (2011). Low temperature removal of inorganic sulfur compounds from mining process waters. Biotechnol. Bioeng. 108, 1251-1259. doi: 10.1002/bit.23057

Lin, H. H., and Liao, Y. C. (2016). Accurate binning of metagenomic contigs via automated clustering sequences using information of genomic signatures and marker genes. Sci. Rep. 6:24175. doi: 10.1038/srep24175

Logan, B. E., Hamelers, B., Rozendal, R., Schroder, U., Keller, J., Freguia, S., et al. (2006). Microbial fuel cells: methodology and technology. Environ. Sci. Technol. 40, 5181-5192. doi: 10.1021/es0605016
Lovley, D. R. (2008). Extracellular electron transfer: wires, capacitors, iron lungs, and more. Geobiology 6, 225-231. doi: 10.1111/j.1472-4669.2008.00148.x

Mangold, S., Valdes, J., Holmes, D. S., and Dopson, M. (2011). Sulfur metabolism in the extreme acidophile Acidithiobacillus caldus. Front. Microbiol. 2:17. doi: 10.3389/fmicb.2011.00017

Marsili, E., Baron, D. B., Shikhare, I. D., Coursolle, D., Gralnick, J. A., and Bond, D. R. (2008). Shewanella secretes flavins that mediate extracellular electron transfer. Proc. Natl. Acad. Sci. U.S.A. 105, 3968-3973. doi: 10.1073/pnas. 0710525105

Matsen, F. A., Kodner, R. B., and Armbrust, E. V. (2010). pplacer: linear time maximum-likelihood and Bayesian phylogenetic placement of sequences onto a fixed reference tree. BMC Bioinformatics 11:538. doi: 10.1186/1471-210511-538

Menzel, P., Ng, K. L., and Krogh, A. (2016). Fast and sensitive taxonomic classification for metagenomics with Kaiju. Nat. Commun. 7:11257. doi: 10. 1038/ncomms 11257

Myers, C. R., and Myers, J. M. (2004). Shewanella oneidensis MR-1 restores menaquinone synthesis to a menaquinone-negative mutant. Appl. Environ. Microbiol. 70, 5415-5425. doi: 10.1128/AEM.70.9.5415-5425.2004

Nancucheo, I., and Johnson, D. B. (2010). Production of glycolic acid by chemolithotrophic iron- and sulfur-oxidizing bacteria and its role in delineating and sustaining acidophilic sulfide mineral-oxidizing consortia. Appl. Environ. Microbiol. 76, 461-467. doi: 10.1128/AEM.01832-09

Nealson, K. H., Belz, A., and McKee, B. (2002). Breathing metals as a way of life: geobiology in action. Antonie Van Leeuwenhoek 81, 215-222. doi: 10.1023/A: 1020518818647

Newman, D. K., and Kolter, R. (2000). A role for excreted quinones in extracellular electron transfer. Nature 405, 94-97. doi: 10.1038/35011098

Ni, G., Christel, S., Roman, P., Wong, Z. L., Bijmans, M. F., and Dopson, M. (2016). Electricity generation from an inorganic sulfur compound containing mining wastewater by acidophilic microorganisms. Res. Microbiol. 167, 568-575. doi: 10.1016/j.resmic.2016.04.010

Nitschke, W., and Bonnefoy, V. (2016). "Energy acquisition in low pH environments," in Acidophiles: Life in Extremely Acidic Environments, eds R. Quatrini and D. B. Johnson (Norfolk, VA: Caister Academic Press), 19-45. doi: 10.21775/9781910190333.02

Osorio, H., Mangold, S., Denis, Y., Nancucheo, I., Johnson, D. B., Bonnefoy, V., et al. (2013). Anaerobic sulfur metabolism coupled to dissimilatory iron reduction in the extremophile Acidithiobacillus ferrooxidans. Appl. Environ. Microbiol. 79, 2172-2181. doi: 10.1128/AEM.03057-12

Quatrini, R., Appia-Ayme, C., Denis, Y., Jedlicki, E., Holmes, D., and Bonnefoy, V. (2009). Extending the models for iron and sulfur oxidation in the extreme acidophile Acidithiobacillus ferrooxidans. BMC Genomics 10:394. doi: 10.1186/ 1471-2164-10-394

Richter, H., Nevin, K. P., Jia, H., Lowy, D. A., Lovley, D. R., and Tender, L. M. (2009). Cyclic voltammetry of biofilms of wild type and mutant Geobacter sulfurreducens on fuel cell anodes indicates possible roles of OmcB, OmcZ, type IV pili, and protons in extracellular electron transfer. Energy Environ. Sci. 2, 506-516. doi: 10.1039/b816647a

Rocca, J. D., Hall, E. K., Lennon, J. T., Evans, S. E., Waldrop, M. P., Cotner, J. B., et al. (2015). Relationships between protein-encoding gene abundance and corresponding process are commonly assumed yet rarely observed. ISME J. 9, 1693-1699. doi: 10.1038/ismej.2014.252

Rohwerder, T., and Sand, W. (2003). The sulfane sulfur of persulfides is the actual substrate of the sulfur-oxidizing enzymes from Acidithiobacillus and Acidiphilium spp. Microbiology 149, 1699-1709. doi: 10.1099/mic.0.26212-0

Rosen, B. P. (1999). Families of arsenic transporters. Trends Microbiol. 7, 207-212. doi: $10.1016 /$ S0966-842X(99)01494-8

Rozendal, R. A., Hamelers, H. V., Rabaey, K., Keller, J., and Buisman, C. J. (2008). Towards practical implementation of bioelectrochemical wastewater treatment. Trends Biotechnol. 26, 450-459. doi: 10.1016/j.tibtech.2008. 04.008

Samartzidou, H., Mehrazin, M., Xu, Z., Benedik, M. J., and Delcour, A. H. (2003). Cadaverine inhibition of porin plays a role in cell survival at acidic $\mathrm{pH}$. J. Bacteriol. 185, 13-19. doi: 10.1128/JB.185.1.13-19.2003

Seemann, T. (2014). Prokka: rapid prokaryotic genome annotation. Bioinformatics 30, 2068-2069. doi: 10.1093/bioinformatics/btu153 
Segata, N., Bornigen, D., Morgan, X. C., and Huttenhower, C. (2013). PhyloPhlAn is a new method for improved phylogenetic and taxonomic placement of microbes. Nat. Commun. 4:2304. doi: 10.1038/ncomms3304

Sharon, I., and Banfield, J. F. (2013). Genomes from metagenomics. Science 342, 1057-1058. doi: 10.1126/science. 1247023

Shi, L., Squier, T. C., Zachara, J. M., and Fredrickson, J. K. (2007). Respiration of metal (hydr)oxides by Shewanella and Geobacter: a key role for multihaem c-type cytochromes. Mol. Microbiol. 65, 12-20. doi: 10.1111/j.1365-2958.2007. 05783.x

Slonczewski, J. L., Fujisawa, M., Dopson, M., and Krulwich, T. A. (2009). Cytoplasmic $\mathrm{pH}$ measurement and homeostasis in bacteria and archaea. $A d v$. Microb. Physiol. 55, 1-79. doi: 10.1016/S0065-2911(09)05501-5

Sola, C., Burgos, M., Plazuelo, A., Toja, J., Plans, M., and Prat, N. (2004). Heavy metal bioaccumulation and macroinvertebrate community changes in a Mediterranean stream affected by acid mine drainage and an accidental spill (Guadiamar River, SW Spain). Sci. Total Environ. 333, 109-126. doi: 10.1016/j. scitotenv.2004.05.011

Stamatakis, A. (2006). RAxML-VI-HPC: maximum likelihood-based phylogenetic analyses with thousands of taxa and mixed models. Bioinformatics 22, 26882690. doi: 10.1093/bioinformatics/btl446

Sulonen, M. L., Kokko, M. E., Lakaniemi, A. M., and Puhakka, J. A. (2015). Electricity generation from tetrathionate in microbial fuel cells by acidophiles. J. Hazard. Mater. 284, 182-189. doi: 10.1016/j.jhazmat.2014.10.045

UniProt Consortium, T. (2018). UniProt: the universal protein knowledgebase. Nucleic Acids Res. 46:2699. doi: 10.1093/nar/gky092

Valdes, J., Quatrini, R., Hallberg, K., Dopson, M., Valenzuela, P. D., and Holmes, D. S. (2009). Draft genome sequence of the extremely acidophilic bacterium Acidithiobacillus caldus ATCC 51756 reveals metabolic versatility in the genus Acidithiobacillus. J. Bacteriol. 191, 5877-5878. doi: 10.1128/JB.00843-09

Vitreschak, A. G., Rodionov, D. A., Mironov, A. A., and Gelfand, M. S. (2002). Regulation of riboflavin biosynthesis and transport genes in bacteria by transcriptional and translational attenuation. Nucleic Acids Res. 30, 3141-3151. doi: $10.1093 /$ nar/gkf433
Wang, Y., Kern, S. E., and Newman, D. K. (2010). Endogenous phenazine antibiotics promote anaerobic survival of Pseudomonas aeruginosa via extracellular electron transfer. J. Bacteriol. 192, 365-369. doi: 10.1128/JB. 01188-09

Wissenbach, U., Kroger, A., and Unden, G. (1990). The specific functions of menaquinone and demethylmenaquinone in anaerobic respiration with fumarate, dimethylsulfoxide, trimethylamine $\mathrm{N}$-oxide and nitrate by Escherichia coli. Arch. Microbiol. 154, 60-66. doi: 10.1007/BF002 49179

Wu, X., Holmfeldt, K., Hubalek, V., Lundin, D., Åström, M., Bertilsson, S., et al. (2015). Microbial metagenomes from three aquifers in the Fennoscandian shield terrestrial deep biosphere reveal metabolic partitioning among populations. ISME J. 10, 1192-1203. doi: 10.1038/ismej. 2015.185

Zammit, C. M., and Watkin, E. L. J. (2016). “Adaptation to extreme acidity and osmotic stress," in Acidophiles: Life in Extremely Acidic Environments, eds R. Quatrini and D. B. Johnson (Poole: Caister Academic Press), 49-62. doi: 10. 21775/9781910190333.03

Zmasek, C. M. (2012). Archaeopteryx: Visualization, Analysis, and Editing of Phylogenetic Trees. Available at: https://sites.google.com/site/cmzmasek/home/ software/archaeopteryx

Conflict of Interest Statement: The authors declare that the research was conducted in the absence of any commercial or financial relationships that could be construed as a potential conflict of interest.

Copyright (C) 2018 Ni, Simone, Palma, Broman, Wu, Turner and Dopson. This is an open-access article distributed under the terms of the Creative Commons Attribution License (CC BY). The use, distribution or reproduction in other forums is permitted, provided the original author(s) and the copyright owner(s) are credited and that the original publication in this journal is cited, in accordance with accepted academic practice. No use, distribution or reproduction is permitted which does not comply with these terms. 